\title{
Composite Implants Coated with Biodegradable Polymers Prevent Stimulating Tumor Progression
}

\author{
N. V. Litviakov ${ }^{1,2, a)}$, S. I. Tverdokhlebov ${ }^{3, b)}$, V. M. Perelmuter ${ }^{1, c)}$, D. E. Kulbakin ${ }^{1, d)}$, \\ E. N. Bolbasov ${ }^{3, e)}$, M. M. Tsyganov ${ }^{1,2, f)}$, A. A. Zheravin ${ }^{1,4, g)}$, V. A. Svetlichnyi ${ }^{2, h)}$, \\ and N. V. Cherdyntseva ${ }^{1,2, i)}$ \\ ${ }^{1}$ Tomsk Cancer Research Institute, Tomsk, 634050 Russia \\ ${ }^{2}$ National Research Tomsk State University, Tomsk, 634050 Russia \\ ${ }^{3}$ National Research Tomsk Polytechnic University, Tomsk, 634050 Russia \\ ${ }^{4}$ Academician E.N. Meshalkin Novosibirsk State Research Institute of Circulation Pathology, Novosibirsk, Russia \\ a)nvlitv72@yandex.ru \\ b) Corresponding author: tverd@tpu.ru \\ c) pvm@ngs.ru \\ d) kulbakin2012@gmail.com \\ e) ebolbasov@gmail.com \\ f) TsyganovMM@yandex.ru \\ g) zheravin2010@yandex.ru \\ h) v_svetlichnyi@bk.ru \\ i) nvch@oncology.tomsk.ru
}

\begin{abstract}
In this experiment we studied oncologic safety of model implants created using the solution blow spinning method with the use of the PURASORB PL-38 polylactic acid polymer and organic mineral filler which was obtained via laser ablation of a solid target made of dibasic calcium phosphate dihydrate. For this purpose the implant was introduced into the area of Wistar rats' iliums, and on day 17 after the surgery the Walker sarcoma was transplanted into the area of the implant. We evaluated the implant's influence on the primary tumor growth, hematogenous and lymphogenous metastasis of the Walker sarcoma. In comparison with sham operated animals the implant group demonstrated significant inhibition of hematogenous metastasis on day 34 after the surgery. The metastasis inhibition index (MII) equaled $94 \%$ and the metastases growth inhibition index (MGII) equaled 83\%. The metastasis frequency of the Walker sarcoma in para aortic lymph nodes in the implant group was not statistically different from the control frequency; there was also no influence of the implant on the primary tumor growth noted. In case of the Walker sarcoma transplantation into the calf and the palmar pad of the ipsilateral limb to the one with the implant in the ilium, we could not note any attraction of tumor cells to the implant area, i.e. stimulation of the Walker sarcoma relapse by the implant. Thus, the research concluded that the studied implant meets the requirements of oncologic safety.
\end{abstract}

\section{INTRODUCTION}

The main goals of today's reconstructive surgery are recovery of formations lost due to cancer or damaged ones, achieving acceptable post-surgery cosmetic and functional results that ensure sufficient quality of a patient's life. The most important anatomic sites are the skullcap, facial skeleton, respiratory passages and chest wall [1]. The enumerated anatomic zones possess a common structure component, the carcass which is different depending on its location, stiffness, configuration and thickness. Reconstruction of such formations involves complex surgical methods including autogenous tissue complex transplantation; duration of such surgery is long and the procedure is multiphase $[1,2]$. At the current stage of reconstructive surgery the need for creating and implementing synthetic and composite tissue replacement materials is becoming more and more apparent. An implant should fit the shape, 
volume, structure, texture of the tissue, and the function of the recovering organ [3]. New tissue replacement systems should combine strong integration potential with longevity in aggressive environment and ability to be filled with medicine to regulate tissue reaction $[3,4]$. Creation and preclinical study of new types of stented composite implants, which are strong and can preserve their shape, and bioactive coating (polymer material) that enhances the integration of an implant are undoubtedly essential and timely.

Reconstructive surgery on oncology patients requires implants to be one more thing —oncologically safe, i.e. it must not possess features that would stimulate relapse of carcinomas in a recipient's tissue that contacts the implant and distant metastases. The problem is long-lasting chronic inflammation around an implant in the area of a removed tumor, it may lead to local relapse and/or stimulate the development of metachronous tumor nidus or distant metastases, i.e. progress of the process [5], thus jeopardizing the results of antitumor treatment. For that matter the stage of preclinical study of implants for oncology patients is essential; it is especially important to study the influence of such implants on the growth and metastasis of experimental tumors.

While completing the 1st stage of a federal program "Research and inventions within priority areas of development of Russian scientific and technological complex for years 2014-2020" (Agreement No. 14.578.21. 0031) a primary screening of integration features was performed for several polymer-based (polylactic acids) bioactive coatings with different organic mineral filling obtained via laser ablation: dibasic calcium phosphate dihydrate $\left(\mathrm{CaHPO}_{4} \cdot 2 \mathrm{H}_{2} 0, \mathrm{DCPD}\right)$ and hydroxyapatite $\left(\mathrm{Ca}_{10}\left(\mathrm{PO}_{4}\right)_{6}(\mathrm{OH})_{2}, \mathrm{HAP}\right)$.

We have determined that the materials obtained via laser ablation of a dicalcium phosphate $\left(\mathrm{CaHPO}_{4}\right)$ solid target is not toxic and provides high adhesion of stem cells [6], which was the reason DCPD was chosen to be studied in terms of oncologic safety.

The aim of the research is to study the influence of composite implants based on biodegradable polymer (polylactic acid) and an organic mineral filling (DCPD) on the growth, metastasis and relapse of the Walker sarcoma in rats.

\title{
MATERIAL AND METHODS
}

\section{Implant}

The experiment involved dummy implants made via "solution blow spinning" (SBS) method [7, 8] based on polylactic acid (PURASORB PL-38) and organic mineral filling obtained via laser ablation of a DCPD solid target. Macroscopically the implants are $10 \times 10 \mathrm{~mm}$ fragments of the material that resembles "filtered paper". The fragments molded easily while being implanted, did not break, demonstrated good adhesion to the surrounding soft tissue (due to the capillary effect).

\begin{abstract}
Animals
40 Wistar male rats weighing 180-200 g and bred by Scientific and Research Institute of Pharmacology and Regenerative Medicine named after E.D. Goldberg were used for the research. The experiments were performed in compliance with humanity principles according to the Rules of work using experimental animals (an act by the Ministry of Healthcare of the USSR from August 12th, 1977) and the principles expressed in the guidelines by the European Community (86/609/EEC) and the Declaration of Helsinki. The animals were kept according to Methodic recommendations of keeping laboratory animals in vivariums of scientific and research institutions and educational institutions (Ruling Documents 3.10.07.02-09) by the Ministry of Agriculture of the Russian Federation with the standard vivarium nutrition and free access to feed and drinking water (tap water with quality in compliance with Sanitary Regulations and Standards 2.1.4.1074-01). The animals were kept under artificial and natural lighting according to the requirements of Construction Rules and Regulations 23.05-95 and Industry Construction Standards 2.10.24.001-04.
\end{abstract}

\section{Implantation of Samples}

The material was implanted under general mixed anesthesia (the dosage is calculated depending on an animal's weight): Zoletil $(5-7 \mathrm{mg} / \mathrm{kg}$ ) and Rometar $(4 \mathrm{mg} / \mathrm{kg})$ intramuscularly. After medication sleep started, we prepared the surgical site: we removed body hair in the area of the animal's pelvis $(3 \times 4 \mathrm{~cm})$ with a Moser $1584-0050 \mathrm{Li}+\mathrm{Pro}$ Mini trimmer (USA). The animals were on the table in the belly-down position. The surgical site was antisepticized 
with a solution of a Septocide R skin antiseptic. The surgical procedures were performed using surgical tools sterilized via autoclaving (Sanitary Regulations and Standards 2.1.3.2630-10).

The ilium was accessed via a 12-20 mm longitudinal cut. Each animal was implanted one sterile sample of the same type with the size of 7-9 $\mathrm{mm}$ in the projection of the ilium. Before the procedure we gained subperiosteal access to the inner surface of the ilium, moreover, we performed scarification of cortical plate with a sharp raspatory. The samples were implanted on the scarified bone surface. The implants were fixed with muscular tissue returned to its original place. The control group animals underwent identical procedures except for the sample implantation.

\section{Design of Research}

4 groups of animals were formed for the research: 1st - the control group (10 animals underwent sham surgery and on the day $17300 \mathrm{mcl}$ of the Walker carcinoma cells suspension were transferred to the surgical site of the ipsilateral paw); 2nd group - experiment 1 (10 animals had a sample implanted and on day 17300 mcl of the Walker carcinoma cells suspension were transferred to the implant site of the ipsilateral paw); 3rd group - experiment 2 (10 animals had a sample implanted and on day $17300 \mathrm{mcl}$ of the Walker carcinoma cells suspension were transferred to the calf of the ipsilateral paw); 4th group - experiment 3 (10 animals had a sample implanted and on day $17100 \mathrm{mcl}$ of the Walker carcinoma cells suspension were transferred to the plantar pad of the ipsilateral paw).

The animals of the control and the 1st groups were evaluated in terms of the implant influence on the primary tumor growth, haematogenous and lymphogenous metastasis of the Walker sarcoma. The influence of the implant on the primary tumor was evaluated by comparing the rates of tumor growth in control and experiment groups. To do so, from day 7 the diameter of the tumor node was being measured in 3 mutually perpendicular directions. Tumor volume was calculated as follows: $V=\pi A B C / 6$, where $A, B, C$ are diameters of the tumor node in 3 mutually perpendicular directions [9].

On day 34 after the surgery or day 17 after the tumor transplantation the rats were euthanized under anesthesia (toxic dosage of Zoletil of 35-40 mg/kg) and the tumors weight was estimated for each animal. After dissection the lungs and par aortic lymph nodes were extracted. The influence of an implant on metastasis of the Walker sarcoma was evaluated based on metastasis frequency, number of metastases in the lungs and their area, moreover, metastasis frequency and metastases weight in the par aortic lymph nodes were evaluated. The metastasis inhibition index was calculated as follows: IIM $=\frac{A_{\text {sham }} B_{\text {sham }}-A_{\text {exp }} B_{\text {exp }}}{A_{\text {sham }} B_{\text {sham }}} \times 100 \%$, where $A$ is the percentage for rats with metastases, $B$ is the number of metastases. The metastases growth inhibition index was evaluated as well based on the total area $(S)$ of metastases according to the following formula: $\mathrm{MGII}=\frac{\sum S_{\text {sham }}-\sum S_{\text {exp }}}{\sum S_{\text {sham }}} \times 100 \%$, where $\sum S_{\text {sham }}$ is the total area of metastases in the control group; $\sum S_{\exp }$ is the total area of metastases in the experiment group [10].

In order to assess the influence of the implant on the Walker sarcoma relapse in rats of the 2nd and 3rd groups, on day 17 after the tumor transplantation we took tissue from the area of the implant for a histological study. By doing so we also determined the presence of tumor cells in the area of the implant which could have come there from the primary site in the calf or a pad of the ipsilateral paw. To gather material for morphological examination we removed fragments of the ilium with the adjacent implant and muscular tissue covering the bone. Samples of the tissue together with the implant were placed in vials with neutral formalin solution. The ratio of the fixing fluid to the sample equaled $20: 1$.

\section{Preparation of Histological Specimen}

The tissue fixed in $10 \%$ formalin for at least 1 day. The samples with bone tissue were decalcified in the mixed formic acid and 10\% formalin solution with the 1:4 ratio. The time for decalcification was 5-7 days. After this the tissue was dehydrated and soaked in paraffin wax in a Leica ASP-300S tissue processor. Further the material was mounted on cassettes. Sections of about 5-7 $\mu \mathrm{m}$ thick were prepared on a Leica RM 2255 rotary microtome, mounted on the slide and dyed with hematoxylin and eosin in a Thermo Gemini AS histology slide stainer. The microscopic evaluation was performed with a Zeiss Axio Scope optical microscope (Germany) with the zoom from $\times 50$ to $\times 100$. 


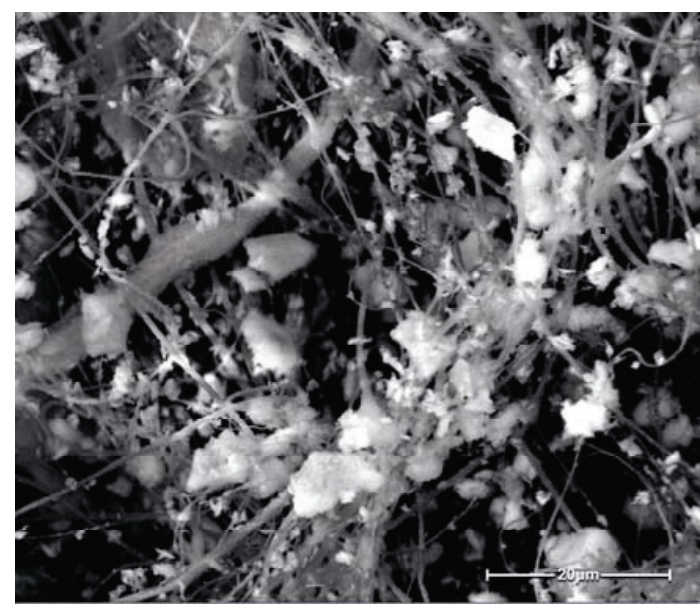

(a)

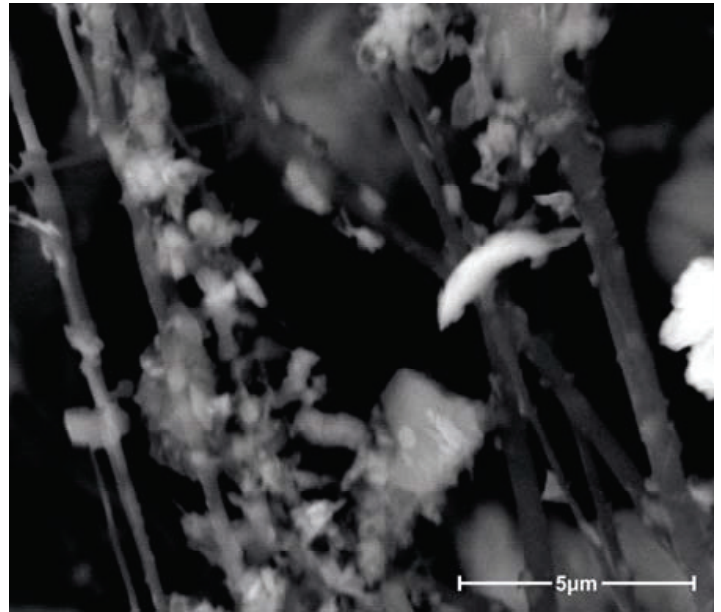

(b)

FIGURE 1. SEM images of implanted materials

\section{Statistical Analysis}

Statistical analyses were performed using STATISTICA 8.0 software (StatSoft, Tulsa, OK, USA). The arithmetic mean value and standard error were calculated for each sample group, and Mann-Whitney U-test was applied to identify the link the volume and weight of primary tumor and lymph node metastases between control and experimental 1st groups. A two-sided p-value was calculated using Fisher's exact test http://vassarstats.net/odds2x2. html. Differences were significant if the $\mathrm{p}$ value was less than 0.05 .

\section{RESULTS AND DISCUSSION}

The images of materials for implantation are presented in the Fig. 1. Formed material (Fig. 1a) possesses fiber pattern with bimodal distribution of the fibers on the diameter, which is common for materials formed via SBS method [11]. Particles of mineral filling in the material are situated on the surface of the fibers. At the same time, big particles are situated in the space between fibers (Fig. 1b).

The formed material possesses high interconnected porosity, which allows normal metabolism and eases the implant's integration with the tissue.

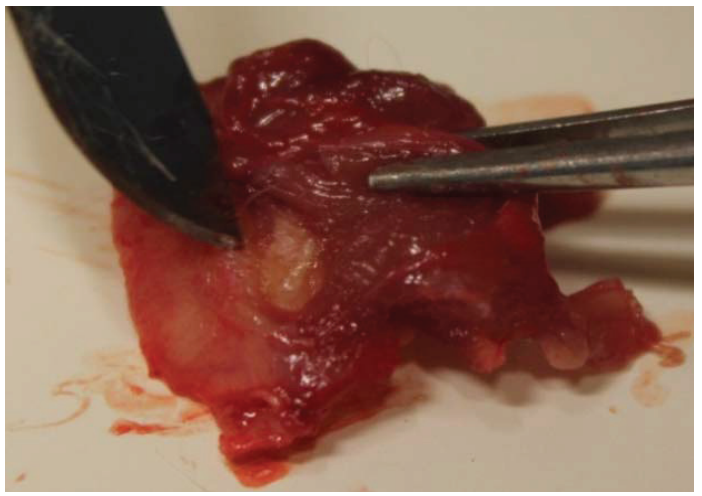

(a)

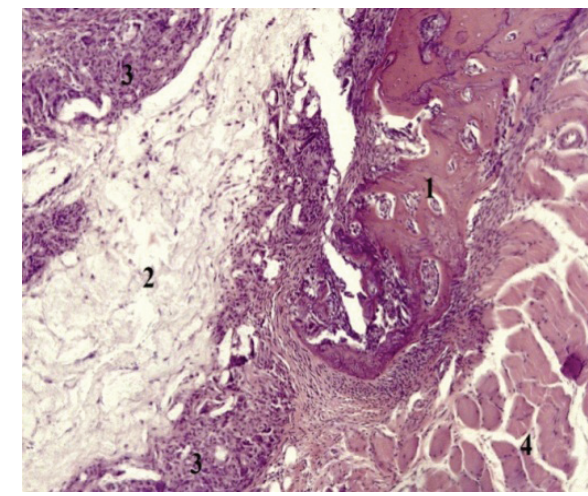

(b)

FIGURE 2. Macroscopic (a) and microscopic (b) preparations of implant region: 1 -bone tissue, 2 - the remaining part of the implant, 3-inflammatory infiltrate, 4-muscular tissue; image zoomed in $\times 100$, decalcification in formic acid, H\&E staining 
TABLE 1. Influence of the implant on the primary Walker sarcoma tumor node in rats

\begin{tabular}{|c|c|c|c|c|c|}
\hline \multicolumn{2}{|c|}{ Day after tumor transplantation } & \multirow{2}{*}{$\begin{array}{c}7 \\
18.6 \pm 2.3\end{array}$} & \multirow{2}{*}{$\begin{array}{c}\mathbf{1 0} \\
35.1 \pm 5.6\end{array}$} & \multirow{2}{*}{$\begin{array}{c}\mathbf{1 3} \\
58.3 \pm 4.3\end{array}$} & \multirow{2}{*}{$\begin{array}{c}\mathbf{1 7} \\
70.8 \pm 4.5\end{array}$} \\
\hline Volume tumor & SHAM & & & & \\
\hline $\mathbf{M} \pm \mathbf{S E}, \mathbf{s m}^{3}$ & Experiment 1 & $17.6 \pm 2.8$ & $38.6 \pm 6.4$ & $60.8 \pm 4.8$ & $67.6 \pm 5.4$ \\
\hline p-value & & 0.886 & 0.663 & 0.845 & 0.532 \\
\hline \multirow{2}{*}{$\begin{array}{l}\text { Weight of rate with } \\
\text { tumor } M \pm S E, g\end{array}$} & SHAM & $289.2 \pm 32.2$ & $309.6 \pm 20.6$ & $326.2 \pm 16.6$ & $334.2 \pm 17.4$ \\
\hline & Experiment 1 & $287.4 \pm 14.3$ & $306.4 \pm 19.4$ & $319.1 \pm 12.7$ & $331.5 \pm 19.3$ \\
\hline p-value & & 0.931 & 0.643 & 0.451 & 0.478 \\
\hline \multirow{2}{*}{$\begin{array}{l}\text { Weight of primary } \\
\text { tumor } M \pm S E, g\end{array}$} & SHAM & - & - & - & $41.3 \pm 3.4$ \\
\hline & Experiment 1 & - & - & - & $41.7 \pm 4.2$ \\
\hline p-value & & - & - & - & 0.786 \\
\hline
\end{tabular}

Macroscopically, on day 15 after the surgery the implant visualized well within the surrounding muscular tissue in the form of a cloudy fragment (Fig. 2a). The implants were fixed to the surrounding muscular tissue and periosteal coverage well due to budding with connective tissue.

The connective tissue capsule was transparent and soft. Microscopic examination on day 15 after the surgery showed that the material was mostly solid, but there was a tendency for microsequestration along the perimeter. There was an expressed giant cell reaction. Macrophage/histiocytic and fibroblastic reactions prevailed. There was a spread of infiltrate onto bone sinuses (Fig. 2b). We also noted the formation of a thicker layer of infiltrate on the side of the muscular tissue and its lesser presence on the side of the bone tissue. Such an observation means there was a positive tendency to integration of the studied samples with the bone tissue.

Thus, by the time of tumor transplantation on day 17 after the surgery the implant was integrated into the surrounding tissue and there was an expressed reaction of the animals' organisms to it in the form of inflammatory giant cell infiltrate with histiocytes and fibroblasts.

The study of the influence on the rate of the primary tumor growth by the implant showed that neither volume of tumor node, nor mass of the body with a tumor, nor mass of the tumor on day 17 after transplantation were statistically different for sham operated animals and the 1st experiment group (Table 1). The animals did not have cachexia during the experiment.

Frequency of the Walker sarcoma metastasis into para aortic lymph nodes for the implant group was not statistically different from the control group. Half of the animals in the control group had metastases in their lymph nodes, as only $30 \%$ of the experiment group animals had those $(\mathrm{p}=0.325 \mathrm{~F}$-test $)$. The average mass of para aortic lymph nodes in the control group equaled $548 \pm 119 \mathrm{mg}$, the same in the experiment group equaled $409 \pm 91 \mathrm{mg}$ $(\mathrm{p}=0.212$, Mann-Whitney U-test).

The influence of the implant on the hematogenous metastasis of the Walker sarcoma was studied. The frequency of metastases in lungs of the control group animals equaled 70\% (7/10) and averaged 4.8 metastases per rat. As for the experiment group, only $2(2 / 10-20 \%)$ animals had metastases in their lungs and the average number of metastases per rat in the group equaled $1(\mathrm{OR}(95 \mathrm{CI})=0.10(0.01-0.83) p=0.035 \mathrm{~F}$-test $)$. Thus, the rats with implants had reduced frequency (lesser percentage of the animals with metastases) and intensity (the average number of lung metastases per rat) of metastasis. The metastasis inhibition index (MII) equaled $94 \%$. The data showed expressed cytotoxic effect, i.e. prevention of metastasis in the group with implants. At the same time this group also showed significant reduction in the total area of metastatic colonies in lungs $\left(27.284 \mathrm{~mm}^{2}\right.$ for the control group and $4.609 \mathrm{~mm}^{2}$ in the experiment group) and high metastasis growth inhibition index (MGII) equaling $83 \%$, which proves cytotoxic effect to be in place - the presence of the implant in an animal's body helps inhibit the growth of already existing metastases. Having an implant "protects" an organism of the tumor bearer from metastases development. Apparently, the expressed inflammatory giant cell reaction to the implant encourages antimetastatic activation of the immune system.

The last stage was to evaluate influence of the implant on the Walker sarcoma relapse. The tumor was transplanted into the ipsilateral paw remotely from the implant (into the calf and a pad).

On day 17 after transplantation tumor samples (Fig. 3a) and implant samples (Fig. 3b) were taken for a microscopic examination. We determined that all 20 animals of the 2 nd and 3rd experiment groups had no tumor cells in the area of the implant. 


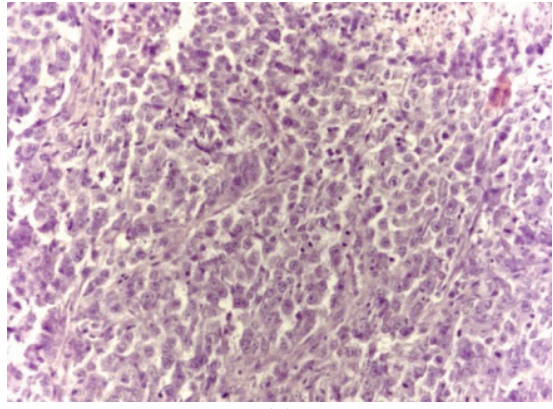

(a)

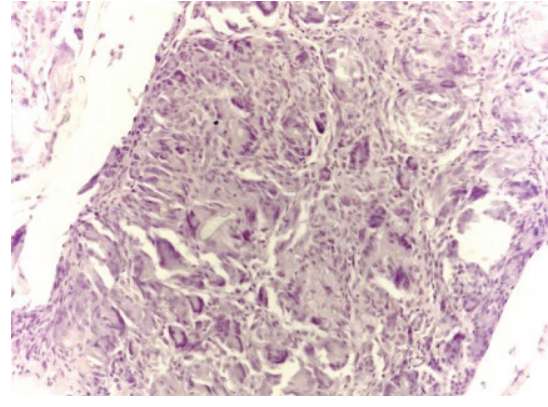

(b)

FIGURE 3. Transfer of tumor into the calf of limb ipsilateral with the implant: (a) the tumor tissue, zoomed in $\times 200$; (b) the inflammatory infiltrate with a big number of giant cells of "foreign bodies", among its cell elements there are no tumor cells, zoomed in $\times 200$, preparation $b$ is prepared after decalcification in formic acid, H\&E stained

Thus, the presence of an implant in the area of the ilium did not attract tumor cells for at least 17 days after tumor transplantation into an ipsilateral limb, i.e. the implant did not stimulate the Walker sarcoma relapse.

The performed research showed that transplantation of composite implants based on biodegradable polymer (polylactic acid) and an organic mineral filling obtained via laser ablation of a solid DCPD target did not influence the primary Walker sarcoma tumor node growth and lymphogenic metastasis, did not stimulate relapse and provided significant antimetastatic effect on hematogenous metastasis. We can conclude that this implant meets the requirements of oncologic safety.

\section{ACKNOWLEDGMENTS}

This work was financially supported by the Ministry of Education and Science of the Russian Federation, Federal Target Program (agreement No. 14.578.21.0031, unique identifier RFMEFI57814X0031).

The study reported in this article was conducted according to accepted ethical guidelines involving research in humans and/or animals and was approved by an appropriate institution or national research organization. The study is compliant with the ethical standards as currently outlined in the Declaration of Helsinki. All individual participants discussed in this study, or for whom any identifying information or image has been presented, have freely given their informed written consent for such information and/or image to be included in the published article.

\section{REFERENCES}

1. S.-J. Shieh, H.-Y. Chiu, J.-C. Yu, S.-C. Pan, S.-T. Tsai, and C.-L. Shen, Plastic Reconstructive Surgery 105(7), 2349-2357 (2000).

2. A. R. Kulkarni, E. D. Sears, D. M. Atisha, and A. K. Alderman, Plastic Reconstructive Surgery 132(3), 534541 (2013).

3. D. Rickert, GMS Current Topics in Otorhinolaryngology, Head and Neck Surgery 8(1), (2009).

4. E. N. Bolbasov, V. V. Guseev, M. V. Dvornichenko, K. A. Nechaev, S. I. Tverdokhlebov, and V. A. Novikov, Bulletin Tomsk Polytechnic University 317(2), 107-111 (2010).

5. V. Perelmuter and V. Manskikh, Biochemistry 77(1), 111-118 (2012).

6. E. N. Bolbasov, I. Lapin, V. Svetlichnyi, Y. Lenivtseva, A. Malashicheva, Y. Malashichev, A. Golovkin, Y. Anissimov, and S. I. Tverdokhlebov, Appl. Surf. Sci. 349, 420-429 (2015).

7. E. S. Medeiros, G. M. Glenn, A. P. Klamczynski, W. J. Orts, and L. H. Mattoso, J. Appl. Polymer Sci. 113(4), 2322-2330 (2009).

8. E. N. Bolbasov, Y. G. Anissimov, A. Pustovoytov, I. A. Khlusov, A. Zaitsev, K. V. Zaitsev, I. Lapin, and S. I. Tverdokhlebov, Mater. Sci. Eng. 40, 32-41 (2014).

9. T. Gudzkova, G. Zhukova, L. Garkavi, M. Sukhanova, O. Evstratova, and T. Barteneva, Bulletin Exp. Biol. Med. 150(5), 659-663 (2011).

10. N. Litviakov, N. Cherdyntseva, V. Belyavskaya, E. Malinovskaya, I. Ilyinykh, and E. Smolyaninov, Voprosy Onkologii 47, 86-89 (2001).

11. A. M. Behrens, B. J. Casey, M. J. Sikorski, K. L. Wu, W. Tutak, A. D. Sandler, and P. Kofinas, ACS Macro Lett. 3(3), 249-254 (2014). 